\title{
A RANDOMIZED CONTROL STUDY TO EVALUATE THE ROLE OF HERBAL IMMUNOMODULATORS IN BOOSTING THE IMMUNITY AND OVERALL HEALTH OF HEALTHCARE WORKERS IN COVID-19 WARDS: AN EXPLORATORY, FEEDBACK CLINICAL STUDY
}

\author{
JAYANTHI CR ${ }^{1}$, AVINASH HR ${ }^{2}$, SWETHA SRIDHAR ${ }^{3}$, AKHILA K ${ }^{1}$, SRIYA SRIDHAR ${ }^{4}$, RAJESH KUMAWAT ${ }^{5 *}$ \\ ${ }^{1}$ Department of Pharmacology, Bangalore Medical College and Research Institute, Bengaluru, Karnataka, India. ${ }^{2}$ Department of General \\ Medicine, Bangalore Medical College and Research Institute, Bengaluru, Karnataka, India. ${ }^{3}$ Department of Dermatology, MVJ Medical \\ College and Research Hospital, Bengaluru, Karnataka, India. ${ }^{4}$ Department of Ophthalmology, RRMCH, Bengaluru, Karnataka, India. \\ ${ }^{5}$ Department of Medical Services and Clinical Development, Himalaya Wellness Company, Bengaluru, Karnataka, India. \\ Email: rajesh.kumawat@himalayawellness.com
}

Received: 13 May 2021, Revised and Accepted: 01 July 2021

ABSTRACT

Objective: The objective of the study was to evaluate the role of the herbal immunomodulators Immusante and Guduchi in boosting the immunity and overall health of healthcare workers assigned to coronavirus disease 2019 (COVID-19) wards.

Methods: An open-label, randomized, prospective, and single center clinical study was conducted among 100 healthcare workers assigned to COVID-19 wards. Eligible subjects (50 in each arm) were randomly assigned to either arm I (Immusante+Guduchi tablets) or arm II (treatment as per the institutional policy). Assessments after 30 days of treatment included respiratory symptoms, Adapted Immune Status Questionnaire (ISQ), improvement in quality of life (Short form 12 [SF-12] Health Survey), and safety and tolerability.

Results: All 100 subjects completed the study as per the protocol. There were no respiratory symptoms in the subjects in arm 1 . However, four subjects (8\%) in arm II reported cough during the study period. A better Immune status improvement was seen through the adapted ISQ in arm I as compared to arm II. In each of the 8 SF-12 Health Survey domains, a statistically significant difference was observed in arm I compared to arm II. A retrospective assessment of COVID-19 infection showed only 8\% of subjects as COVID-19 positive in arm I compared with $26 \%$ of subjects in arm II.

Conclusion: Considering the positive trend observed in this study in terms of ISQ SF-12, and COVID-19 infection rates, the combination of Immusante and Guduchi in healthcare workers at a high risk of contracting COVID-19 infection had a beneficial effect in boosting immunity and overall health.

Keywords: Coronavirus disease 2019, Herbal immunomodulators, Tablet immusante, Tablet Guduchi, Healthcare workers.

(C) 2021 The Authors. Published by Innovare Academic Sciences Pvt Ltd. This is an open access article under the CC BY license (http://creativecommons.org/ licenses/by/4.0/) DOI: http://dx.doi.org/10.22159/ajpcr.2021v14i8.42035. Journal homepage: https://innovareacademics.in/journals/index.php/ajpcr

\section{INTRODUCTION}

The novel coronavirus 2019, severe acute respiratory syndrome coronavirus 2, or coronavirus disease 2019 (COVID-19) was first reported in the city of Wuhan in China and later spread worldwide. It was declared a pandemic on March 11, 2020, by the World Health Organization (WHO) because of its global spread, rapid transmission, and the critical nature of many cases.

Healthcare workers are at a high risk of contracting communicable diseases such as COVID-19 as they perform high-risk activities and spend a lot of time caring for infected patients. Maintaining a healthy immune system is key to preventing infection and disease. Considering the high vulnerability of healthcare workers, it is necessary to provide priority access to testing, treatment, and immunization.

Ayurveda and allied systems of medicine consist of a large repertoire of herbal medicinal agents that may offer a solution to this health care challenge. In recent years, individual herbs as well as polyherbal combinations that help in promoting immunity and respiratory health have undergone rigorous scientific and clinical scrutiny to establish their efficacy and safety. Two examples of potentially helpful herbal agents are Immusante (Prosopis glandulosa $150 \mathrm{mg}$ and Symplocos racemosa $100 \mathrm{mg}$ ) and "Guduchi" (Tinospora cordifolia) tablets and are manufactured at Good Manufacturing Practice certified facilities with adherence to Good Agricultural and Collection Practices (as per the WHO guidelines). Standardization of the production protocol minimizes batch-to-batch variation and assures the safety, efficacy, and quality of these products. Both products have undergone rigorous preclinical and clinical studies for immunotherapeutic support. With this background, the possible roles of Immusante and Guduchi tablets in boosting immunity to COVID-19 are evaluated in this exploratory study.

Immusante is a broad-spectrum immunomodulator developed after decades of research on herbs mentioned in classical Ayurvedic texts. Immusante tablets are recommended as immunotherapeutic support in immunocompromised conditions such as viral and opportunistic infections. These tablets augment immune function, attenuate disease manifestation, and improve resistance to disease. Immusante is known to upregulate interferon gamma [1], fight viral infection, have antioxidant properties, modulate both cellular and humoral immunity, improve the function of neutrophils and macrophages and in turn phagocytosis, augment $\mathrm{CD} 4^{+}$cell count, and promote cytokine gene expression relevant to immunomodulation.

Guduchi, popularly known as "Amrutha" (meaning "nectar" in Sanskrit) in Ayurveda, is an herbal treatment used for fever, infections, and promoting immunity. The immunomodulatory effects of Guduchi have been extensively studied through different experimental models and have also been validated by clinical studies for multiple indications [2]. Guduchi has been found to be effective in relieving the clinical symptoms of allergic rhinitis, cold, and fever, thereby boosts immunity [3]. Guduchi exerts an immunomodulatory effect through macrophage activation and anti-inflammatory effects by inhibiting the expression of inflammatory 
cytokines (such as tumor necrosis factor $-\alpha$ and interleukin $-1 \beta$ ) and decreasing nitric oxide production.

This study was conducted to evaluate the role of the herbal formulations Immusante and Guduchi in boosting the immunity and overall health of healthcare workers assigned to COVID-19 wards.

\section{METHODS}

An open-label, two-armed, randomized, and prospective clinical study was conducted at Victoria Hospital, Bangalore Medical College and Research Institute (Bengaluru, Karnataka, India). The study was conducted in accordance with ethical standards and the Helsinki Declaration after obtaining approval from the ethics committee of the Bangalore Medical College and Research Institute and registration with the Clinical Trials Registry of India (CTRI/2020/07/026579). Informed, signed consent was obtained after a careful explanation of the study from all enrolled subjects.

The inclusion criteria were male and female subjects who were 1860 years old, individuals at a high risk of contracting COVID-19, and community healthcare workers who were assigned to COVID-19 wards. The main exclusion criteria were current active, confirmed, or relapsed COVID-19-positive individuals, pregnant or lactating women, those with a recent history of lung diseases such as asthma and chronic obstructive lung disease, those unable to take oral medications or suffering from ailments related to absorption, those with immunocompromised conditions or taking any immunosuppressant therapy, and subjects suffering from severe and uncontrolled metabolic/endocrinal/cardiac/ renal/hepatic disease, on the basis of history and clinical judgment by the investigator. A randomization list was generated by a biostatistician using online software using the block randomization method with a block size of 4 , and the subjects were randomized into two arms.

After providing informed consent, a total of 100 subjects who fulfilled the eligibility criteria were enrolled and randomized into the two treatment arms in a 1:1 ratio (50 subjects in each arm). Subjects assigned to arm I received Immusante and Guduchi at a dose of 1 oral tablet each, twice daily for 30 days. Subjects in arm II did not receive any treatment to boost immunity. Clinical assessments were performed at the baseline visit (screening day 1) and subjects were followed up and assessed on all subsequent visits, that is, visit 2 (day $7 \pm 3$ days; an optional telephone visit [TO]), visit 3 (day $14 \pm 3$ days or TO), visit 4 (day $30 \pm 3$ days, End of study or T0), and visit 5 (day $45 \pm 3$ days, follow-up visit, or TO). Either visit 4 or visit 5 was required to be an on-site visit. Assessment of immune status based on the Adapted Immune Status Questionnaire (ISQ) was performed on visits 1, 3, 4, and 5.

The Short form 12 (SF-12) Health Survey SF-12 is the abridged practical version of the 36-item Short Form Health Survey (SF-36), which was developed as a tool for measuring health-related quality of life. SF-12 was assessed on visit 4 . The questionnaire contains eight domains comprising of 12 items, covering overall physical and mental health: Physical functioning ( 2 items), role limitations due to physical problems (2 items), body pain (1 item), general health perceptions (1 item), vitality (1 item), social functioning (1 item), role limitations due to emotional problems (2 items), and mental health (2 items). SF-12 summarizes the responses into a physical component summary (PCS) and a mental component summary (MCS).

Statistical analysis of the data generated in this trial was analyzed using GraphPad Prism software v. 6.07 (San Diego, CA, USA). As this was an exploratory feedback study, no formal sample size estimation was done. Means and standard deviations (SD) were calculated for continuous variables, and frequencies and percentages are reported for categorical variables. Normally distributed continuous variables were compared using repeated measures analysis of variance followed by a post hoc test, whereas variables with a non-normal distribution were compared using the nonparametric Friedman test followed by a post hoc test. Within-group analysis was performed by the Friedman test followed by Dunn's multiple comparisons test, and between-group comparisons were assessed using the Mann-Whitney test. The SF-12 Health Survey was analyzed by an unpaired t-test. For comparisons between groups, continuous variables with a normal distribution were compared using the unpaired t-test, whereas variables with a non-normal distribution were compared using the Mann-Whitney test. All statistical tests used a significance level of $\mathrm{p} \leq 0.05$. Two-tailed tests were performed for all analyses.

\section{RESULTS}

\section{Study subjects}

All 100 subjects completed the study according to the study plan, and the study drugs were analyzed for their impact on boosting the immunity and overall health of healthcare workers assigned to COVID-19 wards. Detailed baseline demographics of all subjects in the study are reported in Table 1. The flow diagram of the study as per the Consolidated Standards of Reporting Trials guidelines is depicted in Fig. 1.

\section{Clinical assessment}

At baseline (visit 1), ten subjects in arm I had sore throat, cough, runny nose, nausea, diarrhea, rash, muscle ache, joint ache, loss of appetite, fatigue, or dysgeusia. However, these symptoms subsided by visit 2 , except for sore throat, which was present in one subject $(2 \%)$ and resolved by visit 3 . In arm II, eight subjects had sore throat, cough, chills, nausea, muscle ache, joint ache, loss of appetite, fatigue, or dysgeusia at visit 1 . However, these symptoms subsided by visit 2 , except for cough in one subject (2\%), which resolved by visit 3 (Table 2).

\section{Assessment of immune status based on the ISQ}

Immune status was evaluated in subjects using the ISQ, based on seven parameters (sudden high fever, diarrhea, headache, skin problems, muscle and joint pain, common cold, and cough) (Table 3).

\section{Overall well-being (SF-12 health survey)}

The assessment of overall well-being (SF-12 Health Survey) was conducted at visit 4 (day 30). For the PCS, in arm I, the score was $56.67 \pm 0.64$, compared with $49.76 \pm 5.163$ in arm II, with a statistically significant difference between the two groups $(\mathrm{p}<0.0001)$. For the MCS, in arm I, the score was $61.83 \pm 1.27$ compared with $52.45 \pm 3.997$ in arm II. In addition, a statistically significant difference was observed between the two groups ( $<<0.0001$; Table 4 and Fig. 2).

\section{Incidence of respiratory symptoms during the study}

There was no incidence of respiratory symptoms in any of the subjects in arm I during the study. In arm II, 4 (8\%) subjects complained of cough (Table 5).

Table 1: Summary of demographic characteristics

\begin{tabular}{llll}
\hline Parameter & & Arm I (n=50) & Arm II (n=50) \\
\hline Age (years) & Mean \pm SD & $34.64 \pm 6.37$ & $33.61 \pm 5.85$ \\
& Median & 33.25 & 33.06 \\
& Min-Max & $22.97-58.62$ & $22.32-56.58$ \\
Gender & Male & $21(42 \%)$ & $18(36 \%)$ \\
& Female & $29(58 \%)$ & $32(64 \%)$ \\
Height $(\mathrm{cm})$ & Mean \pm SD & $163.6 \pm 8.97$ & $160.8 \pm 8.19$ \\
& Median & 164 & 160 \\
& Min-Max & $148-180$ & $148-186$ \\
Weight $(\mathrm{kg})$ & Mean \pm SD & $64.88 \pm 11.09$ & $61.36 \pm 11.93$ \\
& Median & 63.5 & 58.5 \\
& Min-Max & $46-90$ & $40-90$ \\
\hline
\end{tabular}

Table 2: No. of subjects with symptoms

\begin{tabular}{lll}
\hline Visits & Arm I (n=50) (\%) & Arm II (n=50) (\%) \\
\hline Visit 1 & $10(20)$ & $8(16)$ \\
Visit 2 & $1(2)$ & $1(2)$ \\
Visit 3 & $0(0)$ & $0(0)$ \\
Visit 4 & $0(0)$ & $0(0)$ \\
Visit 5 & $0(0)$ & $0(0)$ \\
\hline
\end{tabular}




\section{COVID-19 test results}

A reverse-transcription polymerase chain reaction (RT-PCR) test for diagnosing COVID-19 was not part of the study protocol. However, as per the hospital COVID-19 treatment protocol, an RT-PCR test is mandatory for all healthcare workers who are assigned COVID-19 ward duties. The test results of all subjects who participated in this study were therefore collected and evaluated retrospectively. Data were collected that pertain to within 1 month of the completion of the administration of treatment in this study. In this timeframe, only four subjects (8\%) were found to be COVID-19 positive in arm I, while 13 subjects (26\%) were COVID-19 positive in arm II (Table 6, Fig. 3).

\section{Adverse event (AE) profile}

The overall safety profile of both study drugs was found to be good according to the overall assessment. However, in arm I, a total of 5 AEs concerning abdominal pain, a burning sensation in the stomach, and abdominal pain with indigestion were considered as possibly related to the study drugs, and muscle and joint pain and headache, which were not considered to be related to the drugs, were reported in five subjects $(10 \%)$. However, these AEs were mild and resolved on the same day,

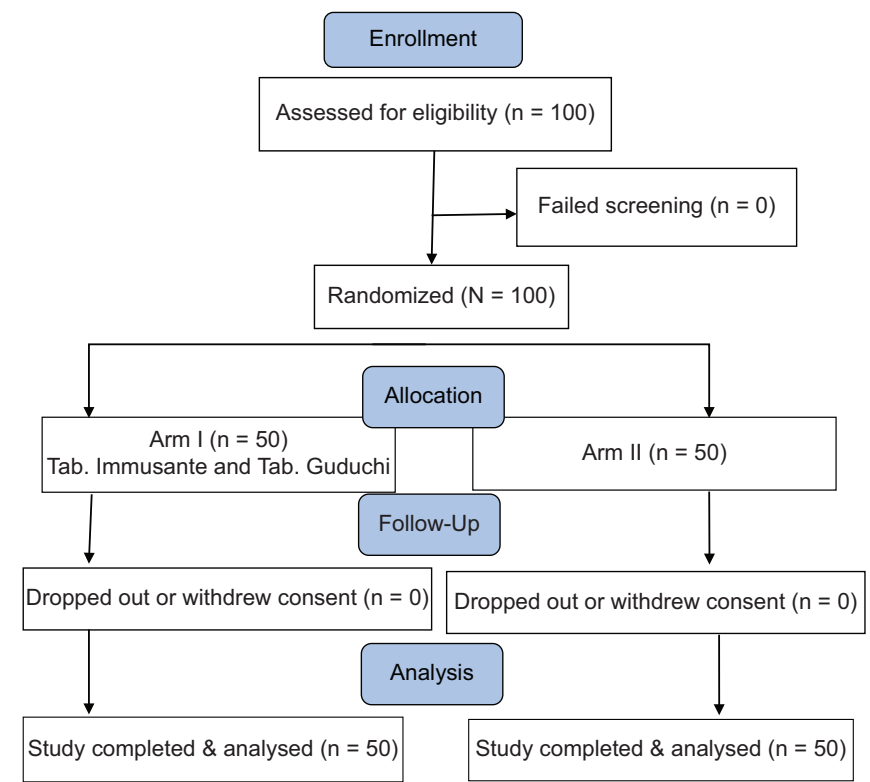

Fig. 1: Flow diagram of the study design as per the Consolidated Standards of Reporting Trials guidelines

Table 3: Immune status questionnaire

\begin{tabular}{|c|c|c|c|}
\hline Visits & & Arm I & Arm II \\
\hline \multirow[t]{3}{*}{ Visit 1} & Mean \pm SD & $0.24 \pm 0.85$ & $0.2 \pm 0.83$ \\
\hline & Median & 0 & 0 \\
\hline & Min-Max & $0-4$ & $0-5$ \\
\hline \multirow[t]{3}{*}{ Visit 3} & Mean \pm SD & $0.3 \pm 0.84$ & $0.2 \pm 0.4$ \\
\hline & Median & 0 & 0 \\
\hline & Min-Max & $0-4$ & $0-1$ \\
\hline \multirow[t]{3}{*}{ Visit 4} & Mean \pm SD & $0.02 \pm 0.14$ & $0.2 \pm 0.53$ \\
\hline & Median & 0 & 0 \\
\hline & Min-Max & $0-1$ & $0-2$ \\
\hline \multirow[t]{3}{*}{ Visit 5} & Mean \pm SD & $0 \pm 0$ & $0.02 \pm 0.14$ \\
\hline & Median & 0 & 0 \\
\hline & Min-Max & $0-0$ & $0-1$ \\
\hline
\end{tabular}

The items were scored on 5 -point scale ranging from 0 to 4 , with the overall score ranging from 0 to 8 , with a higher score indicating a worse immune status. For comparison between groups, the Friedman test followed by Dunn's multiple comparisons test and Mann-Whitney test were used. No statistically significant differences were observed between groups, ISQ: Immune Status Questionnaire. without any sequelae. In arm II, 8 AEs were reported (cough, headache, and muscle and joint pain) by seven subjects (14\%). These AEs were mild and resolved within 1-6 days, without any sequelae. None of the subjects withdrew due to AEs, and no serious AEs were either reported or observed in any of the subjects during the study period. As this was only a feedback study, no laboratory assessments for safety were performed.

All subjects adhered to the prescribed drug dosage and duration and completed the study as per the protocol. There were no clinically

Table 4: SF-12 Health Survey and summary (visit 4)

\begin{tabular}{|c|c|c|c|c|}
\hline \multicolumn{2}{|l|}{ Parameters } & \multirow{2}{*}{$\begin{array}{l}\begin{array}{l}\text { Arm I } \\
(n=50)\end{array} \\
56.47 \pm 0^{*}\end{array}$} & \multirow{2}{*}{$\begin{array}{l}\begin{array}{l}\text { Arm II } \\
(n=50)\end{array} \\
46.51 \pm 7.636\end{array}$} & \multirow{2}{*}{$\begin{array}{l}\text { p-value } \\
\text { (BG) } \\
{ }^{*} \mathrm{p}<0.0001\end{array}$} \\
\hline Physical & Mean \pm SD & & & \\
\hline functioning & Median & 56.47 & 47.88 & \\
\hline & Min-Max & $56.47-56.47$ & $30.7-56.47$ & \\
\hline & $95 \% \mathrm{CI}$ & $56.47,56.47$ & $44.34,48.68$ & \\
\hline Physical role & Mean \pm SD & $56.53 \pm 1.87^{*}$ & $51.37 \pm 4.145$ & ${ }^{*} \mathrm{p}<0.0001$ \\
\hline \multirow[t]{3}{*}{ limitations } & Median & 57.18 & 47.96 & \\
\hline & Min-Max & $47.96-57.18$ & $43.36-57.18$ & \\
\hline & $95 \%$ CI & $56,57.06$ & $50.19,52.55$ & \\
\hline \multirow[t]{4}{*}{ Body pain } & Mean \pm SD & $57.44 \pm 0 *$ & $51.73 \pm 5.11$ & ${ }^{*} \mathrm{p}<0.0001$ \\
\hline & Median & 57.44 & 47.25 & \\
\hline & Min-Max & $57.44-57.44$ & $47.25-57.44$ & \\
\hline & $95 \% \mathrm{CI}$ & $57.44,57.44$ & $50.28,53.19$ & \\
\hline \multirow{4}{*}{$\begin{array}{l}\text { General } \\
\text { health }\end{array}$} & Mean \pm SD & $61.99 \pm 0 *$ & $52.5 \pm 8.668$ & ${ }^{*} \mathrm{p}<0.0001$ \\
\hline & Median & 61.99 & 55.52 & \\
\hline & Min-Max & 61.99-61.99 & $29.65-61.99$ & \\
\hline & $95 \% \mathrm{CI}$ & $61.99,61.99$ & $50.04,54.97$ & \\
\hline \multirow[t]{4}{*}{ Vitality } & Mean \pm SD & $67.48 \pm 1.99 *$ & $54.19 \pm 6.669$ & ${ }^{*} \mathrm{p}<0.0001$ \\
\hline & Median & 67.88 & 57.81 & \\
\hline & Min-Max & $57.81-67.88$ & $27.62-67.88$ & \\
\hline & $95 \% \mathrm{CI}$ & $66.91,68.04$ & $52.29,56.08$ & \\
\hline \multirow{4}{*}{$\begin{array}{l}\text { Social } \\
\text { functioning }\end{array}$} & Mean \pm SD & $56.17 \pm 2^{*}$ & $49.5 \pm 4.675$ & ${ }^{*} \mathrm{p}<0.0001$ \\
\hline & Median & 56.57 & 46.47 & \\
\hline & Min-Max & $46.47-56.57$ & $46.47-56.57$ & \\
\hline & $95 \% \mathrm{CI}$ & $55.6,56.73$ & $48.17,50.83$ & \\
\hline \multirow{4}{*}{$\begin{array}{l}\text { Emotional } \\
\text { role } \\
\text { limitations }\end{array}$} & Mean \pm SD & $55.41 \pm 2.16^{*}$ & $49.26 \pm 4.7$ & ${ }^{*} \mathrm{p}<0.0001$ \\
\hline & Median & 56.08 & 50.49 & \\
\hline & Min-Max & $44.9-56.08$ & $44.9-56.08$ & \\
\hline & $95 \% \mathrm{CI}$ & $54.8,56.02$ & $47.93,50.59$ & \\
\hline \multirow{4}{*}{$\begin{array}{l}\text { Mental } \\
\text { health }\end{array}$} & Mean \pm SD & $64.24 \pm 1.2^{*}$ & $53.07 \pm 3.39$ & ${ }^{*} \mathrm{p}<0.0001$ \\
\hline & Median & 64.48 & 52.34 & \\
\hline & Min-Max & $58.41-64.48$ & $46.27-58.41$ & \\
\hline & $95 \% \mathrm{CI}$ & $63.9,64.58$ & $52.11,54.03$ & \\
\hline \multirow{4}{*}{$\begin{array}{l}\text { Physical } \\
\text { component } \\
\text { score (PCS) }\end{array}$} & Mean \pm SD & $56.67 \pm 0.64^{*}$ & $49.76 \pm 5.163$ & ${ }^{*} \mathrm{p}<0.0001$ \\
\hline & Median & 56.72 & 47.58 & \\
\hline & Min-Max & $55.1-58.87$ & $40.63-61.33$ & \\
\hline & $95 \% \mathrm{CI}$ & $56.48,56.85$ & $48.3,51.23$ & \\
\hline \multirow{4}{*}{$\begin{array}{l}\text { Mental } \\
\text { component } \\
\text { score (MCS) }\end{array}$} & Mean \pm SD & $61.83 \pm 1.27^{*}$ & $52.45 \pm 3.997$ & ${ }^{*} \mathrm{p}<0.0001$ \\
\hline & Median & 62.36 & 52.71 & \\
\hline & Min-Max & $57.51-62.93$ & $42.05-61.44$ & \\
\hline & $95 \% \mathrm{CI}$ & $61.47,62.19$ & $51.31,53.58$ & \\
\hline
\end{tabular}

*Statistical significance in favor of arm I (statistical test: Unpaired $t$ test, level of significance was $P<0.05$. Transformed mean was estimated for the eight SF-12 domains, and the norm-based mean was used for the SF-12 summary measures (PCS and MCS). Statistical test: Unpaired t test, level of significance was $P<0.05$, ns: not significant, BG: between groups. The SF12 data calculates two summary component scores, the PCS and MCS, with eight sub-domains made up of 12 questions in total. Scores range from 0 to 100 , where a zero indicates the poorest health and 100 indicates most healthy. The PCS and MCS combine the 12 responses in such a way that they compare to the national norm, with a mean score of 50.0 and a standard deviation of 10

Table 5: Respiratory symptoms n (\%)

\begin{tabular}{lll}
\hline Symptoms & Arm 1(n= 50) & Arm 2 (n = 50) \\
\hline Cough & $0(0 \%)$ & $4(8 \%)$ \\
\hline
\end{tabular}




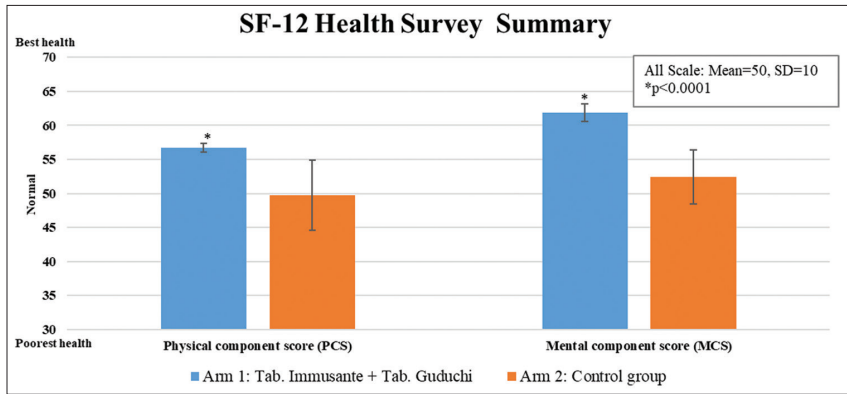

Fig. 2: Short form 12 health survey (PCS and MCS)

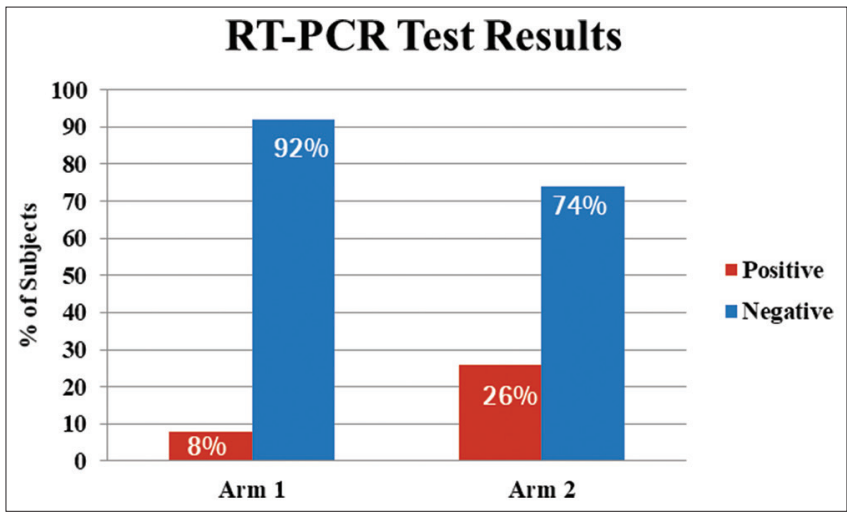

Fig. 3: COVID-19 test results

significant alterations in vital parameters (pulse, respiratory rate, blood pressure, and temperature) or any change in the subjects' medical history or condition. Thus, both the herbal formulations were well tolerated and found to be safe (Table 7).

\section{DISCUSSION}

The COVID-19 pandemic has resulted in severe health consequences. Healthcare workers in particular have additional stressors during patient care such as physical strain, isolation, stringent adherence to infection control procedures, and fears about contracting the infection. Healthcare workers have been found to be socially affected due to their close association with the management of COVID-19 [4].

Tillu et al. enumerated the concepts behind and practices around Ayurveda, that may help manage COVID-19. Integrating the concepts of Ayurveda and yoga with that of modern medicine could be an effective approach toward the overall management of COVID-19, including for the prevention, treatment, and rehabilitation of subjects [5]. Based on their research, Tillu et al. suggested that to strengthen immunity, multiple botanicals that are described in Ayurveda should be used in clinical practice, as they have been in use as medicinal agents for centuries. The authors showed that these botanicals could be considered for the prophylaxis of COVID-19 and as an adjuvant therapy [6]. The selection of products for our study was in line with this concept.

None of the subjects in arm I experienced any respiratory symptoms, but $8 \%$ of the subjects in arm II had a cough. These findings indicate that the administration of Immusante and Guduchi tablets had additional benefits in contributing to the non-occurrence/recurrence of respiratory symptoms during the study.

Immune status was evaluated in subjects by the ISQ, which is a wellvalidated, easily implementable, short, and cost-effective ISQ with applicability in multiple settings, including in clinical practice, research, and self-assessment $[7,8]$. In this study, immune status was rated higher in arm I compared to arm II.
Table 6: COVID-19 test results

\begin{tabular}{lll}
\hline Outcome & Arm I (\%) & Arm II (\%) \\
\hline Positive & $4(8)$ & $13(26)$ \\
Negative & $46(92)$ & $37(74)$ \\
\hline
\end{tabular}

Table 7: AEs

\begin{tabular}{llll}
\hline Description & Total & Arm I (n=50) & Arm II (n=50) \\
\hline Number of AEs & 13 & 5 & 8 \\
Number of subjects & 12 & $5(10 \%)$ & $7(14 \%)$ \\
with AEs-n (\%) & & & \\
AE details & & & 4 \\
$\quad$ Cough & 4 & 0 & 3 \\
$\quad$ Headache & 4 & 1 & - \\
$\quad$ Abdominal pain & 1 & 1 & - \\
$\quad$ Burning sensation in & 1 & 1 & - \\
$\quad$ stomach & & & \\
$\quad$ Abdominal pain with & 1 & 1 & 1 \\
$\quad \begin{array}{l}\text { indigestion } \\
\quad \text { Muscle and joint pain }\end{array}$ & 2 & 1 & \\
\hline AE: Adverse event. & & &
\end{tabular}

In addition, the overall well-being of subjects in this study was assessed through the SF-12 Health Survey, which is widely used and is validated for investigating health-related quality of life in a variety of both acute and chronic conditions. Many studies suggest the results of an SF-12 questionnaire demonstrated significant improvements in physical and mental functioning at the end of the study [9-12]. The outcome in this study is in line with the therapeutic properties of Immusante and Guduchi for enhancing overall well-being.

Ongoing assessment of clinical symptoms is an important aspect of managing the COVID-19 pandemic in terms of prevention as well as ameliorating existing symptoms. As all participants in this study were initially healthy, they were expected to recover from mild symptoms on their own. However, considering the properties of Immusante and Guduchi, these may have helped to boost immunity. The safety findings in this study are in line with the known safety profiles of these products, which have been on the market for many years without any important safety concerns.

In terms of prevention, the prevalence of COVID-19 infection has been reported to be 2,747 cases per 100,000 frontline health care workers compared with 242 cases per 100,000 people in the general community [13]. Another study revealed that among 95\% of infected health care workers who were assigned to a non-COVID-19 facility, $72 \%$ reported exposure to a coworker or patient as the source of infection [14]. Considering the high incidence of COVID-19 infection among healthcare workers, the available data in this study are encouraging. Our results according to COVID-19 tests post-study showed that $8 \%$ of subjects were COVID-19-positive in arm I compared with $26 \%$ of subjects in arm II. Although the sample size in this study is small for a prevention study, our preliminary data on the benefits of Immusante and Guduchi treatment are noteworthy. Further studies with a longer duration of intervention and a larger study population are needed to validate this outcome, which may pose significant benefits to high-risk healthcare workers.

Aside from its limitations, including the inclusion of healthcare professionals in various roles using a variety of protective equipment, the use of open-label drugs, the measurement of results by subjective questionnaires, and the small sample size, this study has some advantages. For instance, this study was conducted at a reputed medical institute (the only dedicated COVID-19 treatment facility in the state at the time this study was conducted) after approval from the ethics committee of the institute and registration in Clinical Trials Registry of India. In addition, the ISQ and SF-12 Health Survey are validated 
questionnaires recommended by the WHO for assessments in this type of study.

\section{CONCLUSION}

This clinical study demonstrates that treatment with a combination of herbal formulations of Immusante and Guduchi tablets in a group of healthcare workers at a high risk of contracting COVID-19 infection had a beneficial effect in boosting their overall health. This is supported by the outcome that no subjects in arm I reported respiratory symptoms compared with four subjects (8\%) who reported cough in arm II. In addition, only $8 \%$ of subjects were diagnosed with COVID-19 in arm I compared with $26 \%$ subjects in arm II. Thus, it can be inferred that a combination of Immusante and Guduchi tablets may be beneficial in protecting high-risk healthcare workers from contracting COVID-19 infection. Subjects in both study arms showed similar status in terms of the ISQ; however, a better trend was observed in arm I compared with that in arm II. In the SF-12 Health Survey results, a statistically significant difference was observed in arm I compared with that in arm II, demonstrating a better health status in subjects who were treated on Immusante and Guduchi tablets compared with that of subjects in the control arm. In addition, there were few AEs and those that were reported were mild in nature and resolved without any sequalae, indicating that these herbal formulations are safe and well tolerated.

Considering the efficacy and safety of our study results, the Immusante and Guduchi combination may be recommended for boosting the immunity and overall health of health care workers (including high-risk personnel who are assigned to COVID-19 wards) for the prevention of respiratory symptoms, protection from contracting COVID-19 infection, and improving their overall physical and mental well-being. Further collection of data in a large population is warranted to corroborate our findings.

\section{ACKNOWLEDGMENT}

We acknowledge Dr Archana R. Shetty for her support in the preparation of this manuscript, Mr Umesh Kumar for data management, and Mr Abdul Rehman for statistical analysis. We extend our thanks to Dr Palaniyamma D. (medical expert) for assisting during the study conceptualization and data interpretation, Dr Soorya Narayan for clinical operation support, and Dr. Sruthi Nambiar for Ayurvedic consultancy in the study. The authors also thank all subjects who participated in this study.

\section{AUTHOR CONTRIBUTIONS}

Dr. C. R. Jayanthi has contributed in conducting clinical study, preparation and review of manuscript. Dr. Avinash HR, Dr. Swetha Sridhar, Dr. Sriya Sridhar and Dr. Akhila K contributed in assisting the clinical study and preparation of manuscript. Dr. Rajesh Kumawat contributed in the revisions and finalization of manuscript.

\section{CONFLICTS OF INTEREST}

Dr. C.R. Jayanthi has received clinical trial support from Himalaya Wellness Company. Dr Rajesh Kumawat is an employee of Himalaya
Wellness Company. The authors declare that the financial interests or personal relationships have not influenced the work reported in this paper. Dr. Avinash H.R, Dr. Swetha Sridhar, Dr. Sriya Sridhar and Dr. Akhila K have no known competing financial interests or personal relationships that could have appeared to influence the work reported in this paper.

\section{FINANCIAL SUPPORT AND SPONSORSHIP}

This study was sponsored and funded by Himalaya Wellness Company (Bengaluru, Karnataka, India).

\section{REFERENCES}

1. Varma RS, Guruprasad KP, Satyamoorthy K, Kumar LM, Babu UV, Patki SP. IM-133N modulates cytokine secretion by RAW264.7 and THP-1 cells. J Immunotoxicol 2016;13:217-25.

2. Kumar R. A randomized study of effect of Tinospora cordifolia in chronic bronchitis patients. IOSR J Dent Med Sci 2018;17:5-10.

3. Geeta S, Kamath MS, Nagendra K, Shenoy RP. A clinical analysis of evaluating the usefulness and efficacy of the ayurvedic drug Tinospora cordifolia in humans. Adv Sci Lett 2017;23:2007-8

4. Menon V, Padhy SK, Pattnaik JI. Stigma and aggression against health care workers in India amidst COVID-19 times: Possible drivers and mitigation strategies. Indian J Psychol Med 2020;42:400-1.

5. Tillu G, Salvi S, Patwardhan B. AYUSH for COVID-19 management. J Ayurveda Integr Med 2020;11:95-6

6. Tillu G, Chaturvedi S, Chopra A, Patwardhan B. Public health approach of Ayurveda and yoga for covid-19 prophylaxis. J Altern Complement Med 2020;26:360-4.

7. Donners AA, Tromp MD, Garssen J, Roth T, Verster JC. Perceived immune status and sleep: A survey among Dutch students. Sleep Disord 2015;2015:721607.

8. Versprille LJ, van de Loo AJ, Mackus M, Arnoldy L, Sulzer TA, Vermeulen SA, et al. Development and validation of the immune status questionnaire (ISQ). Int J Environ Res Public Health 2019;16:4743.

9. Lam CL, Tse EY, Gandek B. Is the standard SF-12 health survey valid and equivalent for a Chinese population? Qual Life Res 2005; 14:539-47.

10. Crocker RL, Hurwitz JT, Grizzle AJ, Abraham I, Rehfeld R, Horwitz R, et al. Real-world evidence from the integrative medicine primary care trial (IMPACT): Assessing patient-reported outcomes at baseline and 12-month follow-up. Evid Based Complement Altern Med 2019;2019:8595409.

11. Scherwitz LW, Cantwell M, McHenry P, Wood C, Stewart W. A descriptive analysis of an integrative medicine clinic. J Altern Complement Med 2004;10:651-9.

12. Kligler B, Homel P, Blank AE, Kenney J, Levenson H, Merrell W. Randomized trial of the effect of an integrative medicine approach to the management of asthma in adults on diseaserelated quality of life and pulmonary function. Altern Ther Health Med 2011;17:10-5

13. Nguyen LH, Drew DA, Graham MS, Joshi AD, Guo CG, Ma W, et al. Risk of COVID-19 among front-line health-care workers and the general community: A prospective cohort study. Lancet Public Health 2020;5:e475-83.

14. Alajmi J, Jeremijenko AM, Abraham JC, Alishaq M, Concepcion EG, Butt AA, et al. COVID-19 infection among healthcare workers in a national healthcare system: The Qatar experience. Int J Infect Dis 2020;100:386-9. 